\title{
Integration of Energy Storage in Distribution Grids
}

\author{
Frederik Geth, Student Member, IEEE, Jeroen Tant, Student Member, IEEE, Edwin Haesen, Member, IEEE, \\ Johan Driesen, Member, IEEE, and Ronnie Belmans, Fellow, IEEE
}

\begin{abstract}
Electrical energy storage services can bring benefit to multiple stakeholders in the distribution grid. Energy storage owners maximize their profit on an external energy market. This can cause a conflict with the distribution system operator because a grid is designed in terms of peak power, not energy. The subject of this paper is a optimization method for the siting and sizing of energy storage in distribution grids. The optimization is implemented multi-objective as to visualize the trade-off's between storage stakeholders and distribution system operators.
\end{abstract}

Index Terms-Battery storage plants, Power distribution planning, Distributed energy resources, Multi-objective optimization methods, Load flow analysis

\section{INTRODUCTION}

In light of recent frameworks on climate change mitigation, an increasing penetration of Distributed Energy Resources (DER) is to be expected. For example in Europe multiple objectives were set to be met by $2020: 20 \%$ reduction in energy consumption, $20 \%$ market share of renewable energy and $10 \%$ sustainable bio-fuels for transport [1]. There are incentives in place for residential users to partake in the production of this sustainable electricity. Combined heat and power, photovoltaic and wind generation are made economically viable by means of feed-in tariffs, subsidies or renewable energy certificates.

Aforementioned technologies have an inherently variable and difficult to predict power output as they all are in some degree dependent on the weather. With increasing penetration, optimal integration will require some degree of matching of the generation profiles, the load profiles and the transport of electricity. Wind and photovoltaic DERs have a high peak-toaverage-power ratio. This makes transport more inefficient as line losses become progressively higher with power. Also, the supply of electricity and the demand of residential users is likely to be badly synchronized in case of random integration. Comparing for example the generation by photovoltaic to residential load: photovoltaic generation during the day will peak while people are at work and residential load is low.

The integration of electricity storage in a distribution grid could mitigate some of the problems of a high penetration of DER. Generation and load profiles could be matched and line losses can be controlled. Voltage can be supported by active power injection which can defer otherwise needed grid improvements.

Optimal siting and sizing of stochastic and controllable DER using genetic optimization was proposed by [2]. The optimization performed is multi-objective because more insight is needed in the compromises between the objectives of DER

F. Geth, J. Tant, E. Haesen, J. Driesen and R. Belmans are with the Research Group ELECTA, Department of Electrical Engineering, K.U. Leuven, Leuven 3001, Belgium (e-mail: frederik.geth@esat.kuleuven.be) stakeholders and DSOs. The revenue of a DER stakeholder depends on the energy produced, whereas the Distribution System Operator (DSO) designs his grid in terms of peak power. DER have the potential to lower line losses in the grid but where supply and demand are badly matched, grid improvements might be necessary in case of sustained passive grid operation. With multi-objective analysis the trade-offs between objectives can be visualized.

This paper is structured in the following manner. Firstly, different storage technologies are assessed on usability in a distribution grid and DER integration potential. Secondly, an Optimal Power Flow (OPF) algorithm with adaptations for storage integration analysis is proposed. Finally, the siting and sizing of the battery storage is optimized using a genetic algorithm adapted for this problem. Both elements of the proposed method are illustrated with an example distribution grid with DERs.

\section{Means of Energy Storage}

Energy storage in distribution grids is primarily bounded by a two factors: discharge time and power rating. A large set of storage technologies is available for support of DERs; among others: Battery Energy Storage (BES), flow batteries, fuel cells, flywheels. They all can offer the necessary ratings for power and discharge time, however there is much variation in viability. Comparisons can be found in literature [3], [4]. For the sake of simplicity, a general type of battery is considered in this research. Batteries allows for easy scaling in terms of both capacity and power rating. Specific battery technologies can be simulated and compared but this is not within the scope of this research.

The total cost for the storage system include costs for the batteries, the grid interface and the efficiency of the storage. Battery costs depend on the required ratio of power-to-energy, which depends on the battery chemistry. Investment for the power electronics to control the energy flow depends on the power rating. Using a battery incurs efficiency losses for charging, discharging and stand-by. Energy content in a lot of cases is restricted to a chosen limit on depth of discharge because cycles of high discharge can cause premature aging of the cells. Consequently, energy content in each time step of the optimization is limited by a minimum and maximum state of charge and evolves from previous time steps by the mechanisms of self-discharging, charging and discharging.

$$
\begin{aligned}
& \forall i \in B, \forall j \in 1 \ldots t^{\max }: \\
& E_{i}^{\min } \leq E_{i}^{0} \eta_{\mathrm{sb}}+\Delta t \sum_{j=1}^{t}\left(X_{\mathrm{BESc}, i}^{j} \eta_{\mathrm{charg}}+\frac{X_{\mathrm{BESd}, i}^{j}}{\eta_{\mathrm{disch}}}\right) \eta_{\mathrm{sb}} \leq E_{i}^{\max }
\end{aligned}
$$




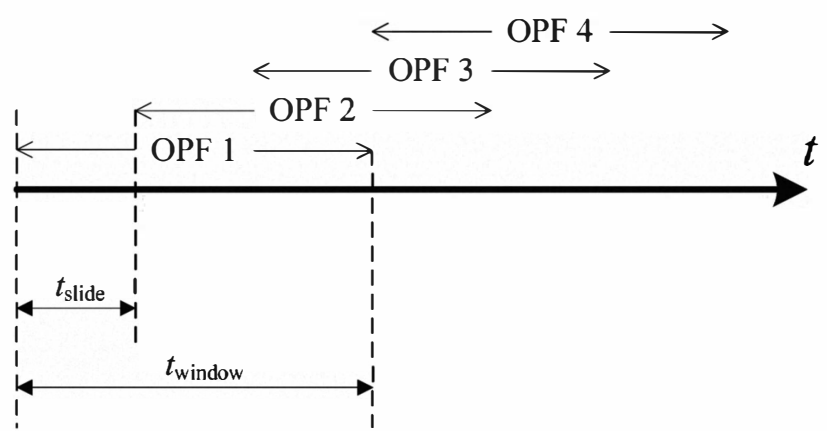

Fig. 1. Sequential OPF formulations

In Formula 1, $X_{\mathrm{BES}, i}^{j}$ represents the power injection in the grid of the BES unit connected at node $i$ at time interval $j$, $E_{i}^{j}$ the corresponding energy content of the BES, $\mathrm{N}$ the set of nodes for which the voltage constraint is set and $\mathrm{B}$ the set of nodes at which a BES unit is connected. $\eta_{\mathrm{sb}}, \eta_{\mathrm{charg}}$ and $\eta_{\text {disch }}$ are respectively the stand-by, charge and discharge efficiencies.

\section{OPTIMAL POWER FLOW WITH BES}

\section{A. OPF algorithm}

In order to apply a multi-objective optimization for BES siting and sizing, a model is needed which emulates the distribution grid behavior for a candidate BES topology. The proposed method assumes that all storage units aim at maximizing profit on a power exchange (PX) and that the storage unit is too small to affect energy prices, resulting in the general trend of 'buying low and selling high' [5]. A fundamental difference with other OPF algorithms used for DER dispatch and curtailment, is that OPF for BES operation encompasses multiple time steps at once. In case of storage, the individual time steps are no longer independent. The algorithm as formulated by [6] is:

$$
\begin{array}{lll}
\min & \Delta t \sum_{j=1}^{t^{\max }} \sum_{i \in B} C_{\mathrm{PX}}^{j} X_{\mathrm{BES}, i}^{j} & \\
\text { s.t. } & U^{\min } \leq\left|U_{S}^{j}\right| \leq U^{\max } & \forall s \in N, \forall j=1 \ldots t^{\max } \\
& -P_{\mathrm{BES}, i} \leq X_{\mathrm{BES}, i}^{j} \leq P_{\mathrm{BES}, i} & \forall i \in B, \forall j=1 \ldots t^{\max } \\
& E_{i}^{0}=E_{i}^{t^{\max }}=E_{i}^{\min }+\frac{1}{2}\left(E_{i}^{\max }-\right. & \left.E_{i}^{\min }\right) \\
& \text { Equation (1) }
\end{array}
$$

In this Formula, the meaning of the symbols is the same as in Formula 1 and $C_{\mathrm{PX}}$ is added as a power exchange price curve. The solution to this optimization problem maximizes the aggregated revenue of all BES units. For each time step $j$, a backward/forward sweep distribution power flow algorithm [7] is applied to calculate the nodal voltage values $U_{\text {node }}^{j}$, based on the nodal power $S_{\text {node }}^{j}$ values. Time profiles for load and distributed generation of all nodes are given as input to the algorithm. Voltage constraints are set on some nodes to guarantee acceptable voltage quality and power constraints are set for each BES. By setting the state of charge for BES at

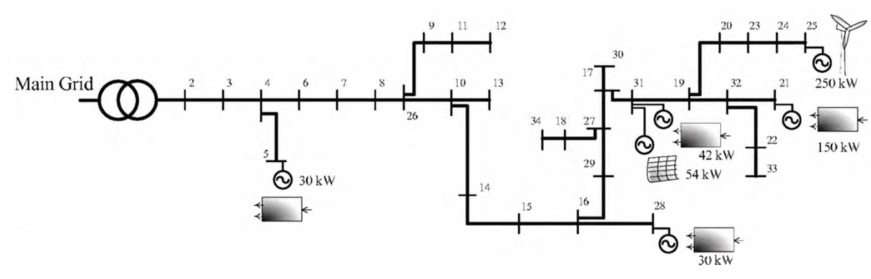

Fig. 2. IEEE 34-bus system with generation units.

$50 \%$ at the boundaries of the time span, continuity is assured with events preceding and following the current time span.

For each evaluation of a candidate topology, the OPF algorithm is executed. To lower the computational burden, the time span can be decreased by using sequential OPFs with a sliding window. This method is visualized in Figure 1. $E_{i}^{0}$ needs to be adjusted in each OPF, based on the previous run. The extent in which $t^{\max }-t^{1}$ can be lowered depends on $E_{i}^{\max } / P_{\mathrm{BES}, i}$. Also will $t_{\text {slide }}$ and $t_{\text {window }}$ need to be chosen so that a repeated bias towards $\frac{1}{2}\left(E_{i}^{\max }-E_{i}^{\min }\right)$ is avoided.

\section{B. Test case}

As a test case, the IEEE 34-bus system [8] at $24 \mathrm{kV}$ in Figure 2 is considered with a $2.3 \mathrm{MW}$ peak load and low level (22\%) of DER penetration. Multiple types of loads are connected: CHP, photovoltaic and wind DERs. Loads in this grid are not actively controlled. An example OPF for electrical energy storage in the IEEE 34-bus system is illustrated in Figure 3. BES units are connected at nodes 5, 10, 15, 20, 25 and 30. The units have an efficiency of $\eta_{\text {charg }}=\eta_{\text {disch }}=0.9$ and $\eta_{\mathrm{sb}}=0.99$ - as a Li-Ion battery would have [9] - with a power rating of $100 \mathrm{~kW}$ and maximum energy content of $500 \mathrm{kWh}$. The multiple graphs in Figure 3 show, from top to bottom: the voltages at the BES buses with and without BES units, the power injected by and the energy content of each BES, aggregated load and power and a given power exchange curve. Figure 4 shows the OPF for the same conditions as the one just described, but without possibility of selling and buying energy on the PX because the price is fixed.

The voltage profile with BES shows much more variability than without BES. The voltage however is in magnitude constraint to a minimum and maximum. The variation is a result of the profit maximization on the PX. When prices rise, energy is delivered to the grid, e.g. around 3-4AM. The price variations are reflected in the injection power which in term influence the voltage level. At times when prices fall, e.g. 12-2PM, the BES will charge and the resulting voltage level will be lower than without BES. However, around 5-6PM, the voltage level can now, with the help of BES, be maintained within the specified margin. The BES will all discharge to maintain the minimum voltage level. This test case shows already some insight in the objectives of storage stakeholders and DSOs: profit maximization must consider grid limitations.

The other way around is shown in Figure 4. With a constant price curve - no arbitrage of energy - will still lead to cycling of the BES because of voltage constraints. This simulation determines the cost of ancillary services by a dedicated BES. 

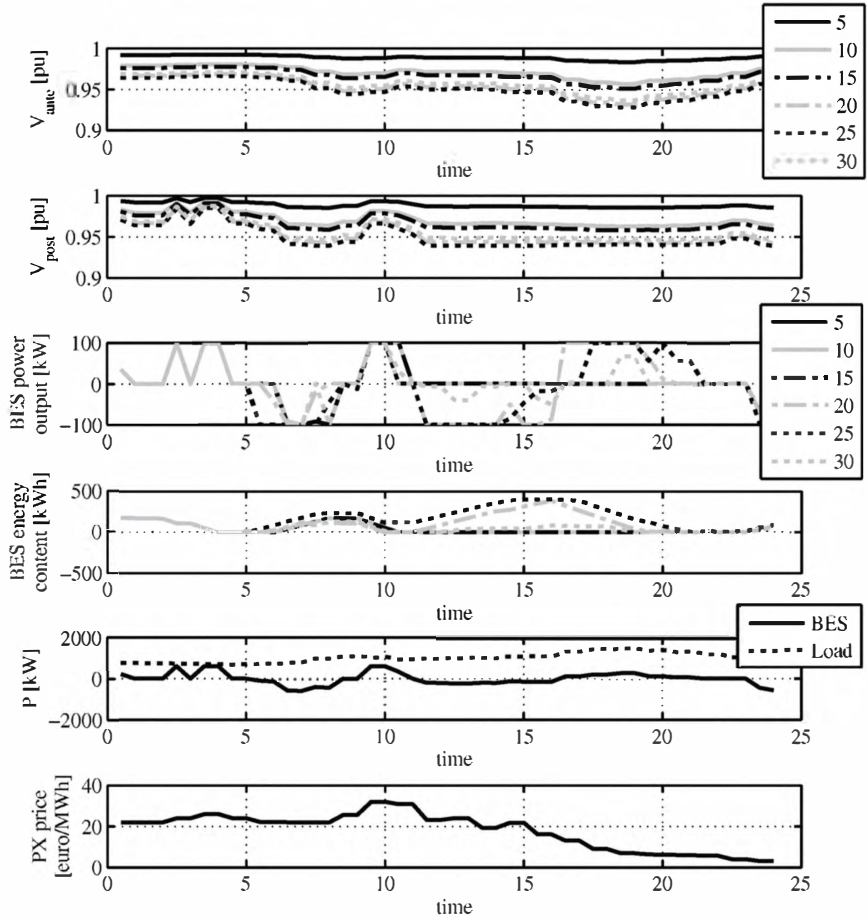

Fig. 3. Results of the OPF outcome for six BES units in the IEEE 34 bus system, showing the voltages at the BES buses with and without BES, the power injection and energy content of the BES units, aggregated load and power injection and the PX curve in which revenue maximization is pursued.
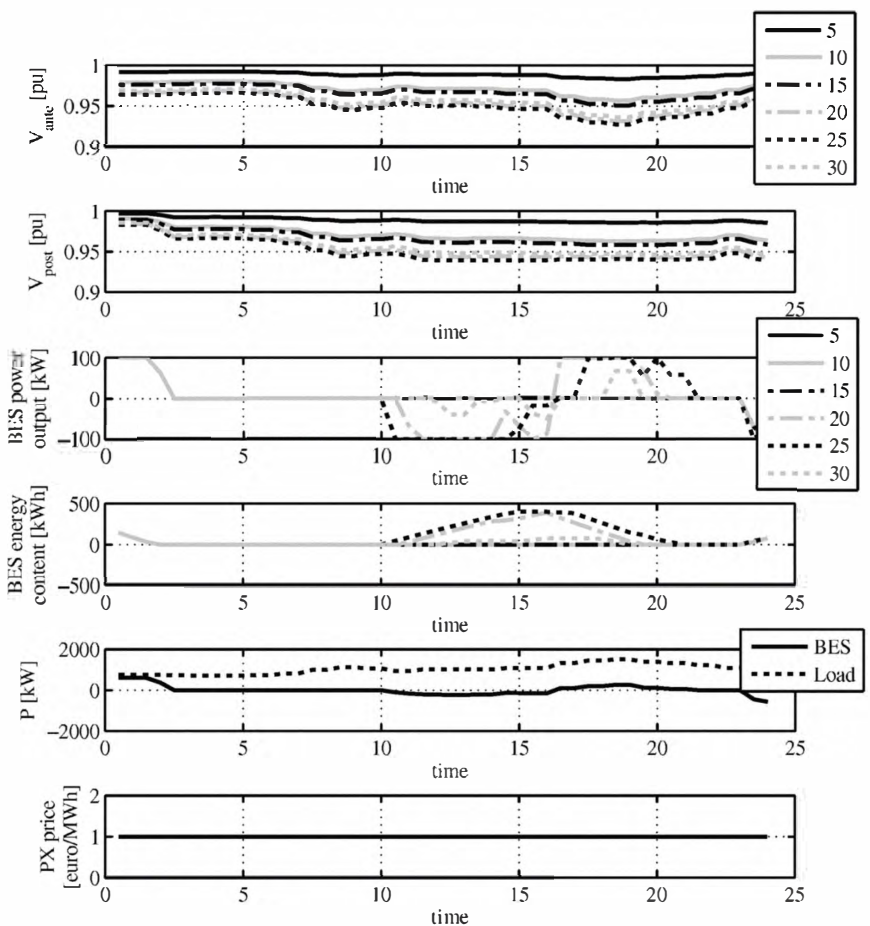

Fig. 4. Results of the OPF outcome for six BES units in the IEEE 34 bus system, showing the voltages at the BES buses with and without BES, the power injection and energy content of the BES units, aggregated load and power injection when no revenue maximization is pursued.

\section{Multi-OBJECTIVE OptimizATION FOR Siting AND SIZING OF BES UNITS}

In the previous section, a storage model was implemented and optimal power flow could be calculated for a given situation. It was not yet decided where the storage will be localized and what the power and energy rating will be. In this paper, the multi-objective method for optimal siting and sizing of DER described in [2] is adapted and used to determine the optimal placement of BES units in the grid. Candidate topologies are evaluated by using the power flow calculations of the previous section.

When the solution of a multi-objective optimization problem is considered, the objective functions generally can't be all optimal at once. There usually is a trade-off between some of the objectives. These trade-offs can be visualized with a set of Pareto-optimal points, forming the Pareto-front. A solution is Pareto optimal if no improvement can be made in one of the objectives without worsening another objective.

The task of a multi-objective optimization algorithm is to find a set of non-dominated solutions that belong to the Pareto front. As in [2], the multi-objective genetic algorithm SPEA-II [10] is used. The decision space is formed by all possible BES topologies in the grid. A candidate topology is represented with a chromosome in the genetic algorithm. This chromosome is formed by two discrete numbers for each node where a BES unit can be connected to. The two discrete numbers represent the power output and energy content ratings of a BES unit at the corresponding node.

The optimal integration of BES units is analyzed with respect to five objectives, i.e.

1) minimization of the total installed BES rated power $[\mathrm{kW}]$

2) minimization of the total installed BES rated energy $[\mathrm{kWh}]$

3) minimization of the probability on voltage deviations larger than $6 \%[\%]$

4) maximization of the total BES revenue on a power; exchange market [euro/year];

5) minimization of the opportunity loss in BES revenue due to the requirement to deliver ancillary services if possible [euro/year].

For objective 4, the OPF of the previous section is applied. In order to account for stochastic variations, different sets of load, generation and power exchange profiles are used in each generation of the SPEA-II cycle. Short profiles of 4 days, each consisting of 48 half hour steps, are evaluated for each candidate BES topology. The resulting aggregated BES revenue is indicated as $R_{\mathrm{PX}}$, grid. When no feasible $\mathrm{BES}$ operation solution exists, no BES dispatch occurs during this day and as such $R_{\mathrm{PX}}$, grid $=0$. Additionally to the OPF a simple BES revenue maximization is applied in which no grid constraints are considered, resulting in a BES remuneration $R_{\mathrm{PX}}$. The difference between both profits is a measure for the required remuneration of $\mathrm{BES}$ ancillary services (AS) $R_{\mathrm{AS}}$, or vice versa the opportunity loss due to grid constraints, and is taken in the optimization problem as objective 5 .

$$
R_{\mathrm{AS}}=R_{\mathrm{PX}}-R_{\mathrm{PX}, \text { grid }}
$$



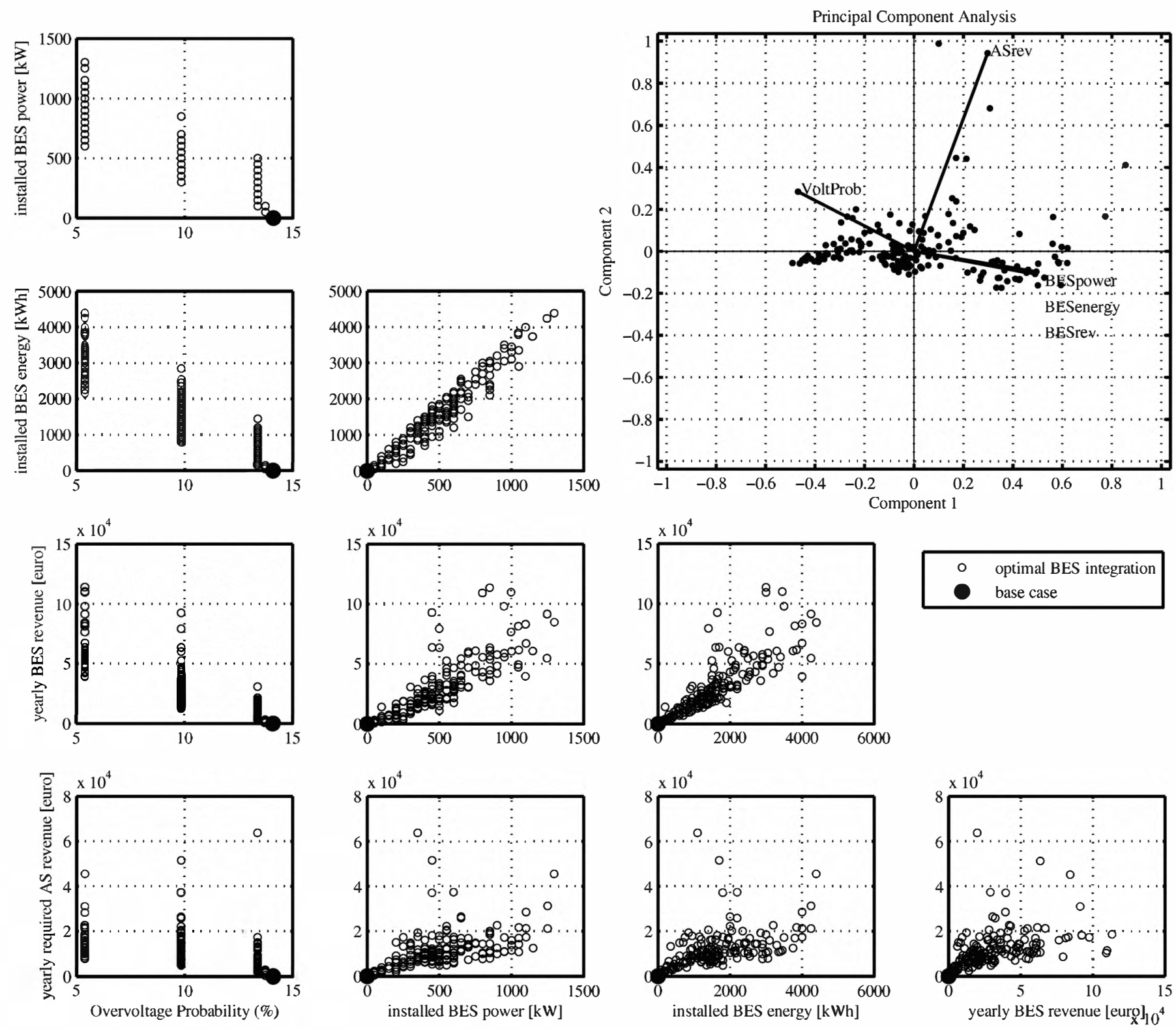

Fig. 5. Pareto optimal set for the integration of BES units in the IEEE 34-bus system. 


\section{RESULTS}

The multi-objective analysis is performed on the test case of section III-B. BES units can be connected at varying locations in the distribution grid. There are two decision variables for each possible location, being the power output in a discrete range of $50 \mathrm{~kW}$ up to $500 \mathrm{~kW}$ and the rated energy content in discrete steps of 1 up to 5 hours. The result of the corresponding multi-objective optimization is given in Figure 5 as a set of Pareto optimal points. The visualization shows the trade-offs between each set of two objectives. A principal component analysis (PCA) is given as well. In this PCA, the 5-dimensional result space is aligned with the two directions of largest variance and projected on a two-dimensional plot. A small angle between two objective vectors indicates a strong linear correlation. Care must be taken when two vectors are aligned in the two-dimensional plot, as they can form an angle in the hidden dimensions.Furthermore, linear correlations are assumed in the PCA. Therefore, it is advisable to use all available plots to gain insights in the trade-offs.

There is a strong linear correlation between installed BES power and energy ratings. The voltage violation probability vector lays in opposite direction. Installing more BES lowers the probability of voltage violation. The relations between total installed BES ratings, BES revenue and AS revenue are non-linear and less trivial. As an example, a 1000kW BES integration guarantees the lowest voltage violation probability while BES revenue and AS revenue opportunity loss are at a local maximum and minimum.

\section{CONCLUSION}

The study of [11] assessed the feasibility of electricity storage from the viewpoint of BES owners. As shown, DER stakeholders and DSOs can have conflicting interests. This research quantified some of the trade-offs involved for both. Siting and sizing of electricity storage was optimized stochastically for multiple objectives in a IEEE 34-bus grid.

Energy storage is often cited as a facilitating and sometimes essential component for the integration of stochastic generation and deferral of grid reinforcements. The BES units are operated to maximize revenue on an external energy market while delivering ancillary services for voltage control and congestion management by means of a central OPF when possible. By comparison with revenue maximization in absence of grid problems, the value of these ancillary services, or vice versa the opportunity loss due to grid constraints, can be assessed. Optimizing distribution grids with the addition of $\mathrm{BES}$ is more complex than without because of time step dependencies.

Next to decentralized generation and electrical energy storage, also active loads are part of DER. The OPF for BES operation can be used as well to dispatch flexible loads in case of grid problems. This requires a different set of constraints, e.g. with a required total energy consumption for each active load at a specific time. When including this in the DER optimization the goal most likely is not to optimize the location of new active loads, rather than to asses the optimal connection points for activation of passive loads.
The test case for the newly proposed method showed the potential of optimal storage integration as a means of controlling grid limitations. In view of a smart grid context one can look into Community Energy Storage (CES). These are small energy storage units, in the order of $25 \mathrm{~kW}$ for a few hours. One module could provide energy storage for a number of houses. Such units would be connected to the low voltage grid where large voltage variations are expected with a high penetration of DER. Preferably existing distribution grids could be analyzed.

Integrating demand side management in the newly proposed method and simulating a realistic low voltage grid with CES are opportunities for future research.

\section{ACKNOWLEDGMENT}

The authors would like to thank A. Alarcon-Rodriguez and G. Ault from the University of Strathclyde for their support in this research.

\section{REFERENCES}

[1] European Commission. (2008, January 21) Proposal for a Directive of the European Parliament and of the Council on the promotion of the use of energy from renewable sources. (Last checked July 2009). [Online]. Available: http://eur-lex.europa.eu/LexUriServ/ LexUriServ.do?uri=CELEX:52008PC0019:EN:NOT

[2] A. Alarcon-Rodriguez, E. Haesen, G. Ault, J. Driesen, and R. Belmans, "Multi-objective planning framework for stochastic and controllable distributed energy resources," IET Journal on Renewable Power Generation, vol. 3, no. 2, pp. 227-238, June 2009.

[3] H. Ibrahim, A. Ilinca, and J. Perron, "Energy storage systemscharacteristics and comparisons," Renewable and Sustainable Energy Reviews, vol. 12, no. 5, pp. 1221-1250, 2008.

[4] L. Mears, H. Gotschall, and H. Kamath, "Handbook of energy storage for transmission or distribution applications," Electric Power Research Institute, Tech. Rep., 2003.

[5] R. Sioshansi, P. Denholm, T. Jenkin, and J. Weiss, "Estimating the value of electricity storage in PJM: Arbitrage and some welfare effects," Energy Economics, vol. 31, no. 2, pp. 269-277, March 2009.

[6] E. Haesen, "Multi-objective optimization of the integration of stochastic distributed energy resources in electricity grids," Ph.D. dissertation, K.U.Leuven, 2009.

[7] R. Ciric, A. Padilha, and L. Ochoa, "Power flow in four-wire distribution networks - general approach," IEEE Power Engineering Society General Meeting, 2003, vol. 18, no. 4, pp. 1283-1290, November 2003.

[8] W. Kersting, "Radial distribution test feeders," IEEE Transactions on Power Systems, vol. 6, no. 3, pp. 975-985, August 1991.

[9] B. Lunz, "Evolution of batteries and charging concepts," Seminar Clean Mobility, KHLim, Diepenbeek, Belgium, 16 December 2008.

[10] E. Zitzler, M. Laumanns, and L. Thiele, "SPEA2: Improving the Strength Pareto Evolutionary Algorithm," ETH Zurich, Tech. Rep., 2001.

[11] F. Nieuwenhout, K. Visscher, G. Schaeffer, M. Hommelberg, and J. Kester, "Feasibility of distributed electricity storage," International Journal of Distributed Energy Resources, vol. 2, no. 4, December 2006. 


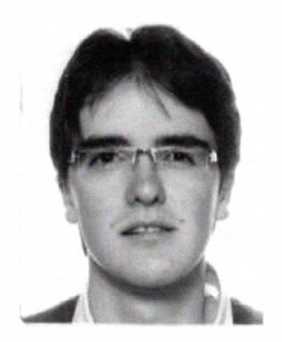

Frederik Geth (S'10) received the M.Sc. degree in electrical engineering from the Katholieke Universiteit Leuven (K.U.Leuven), Leuven, Belgium, in 2009. Currently, he is working as a research assistant with the division ESAT-ELECTA. His research interests include optimal storage integration in distribution grids, batteries for (hybrid) electrical vehicles and controlling the impact of the charging currents of (hybrid) electrical vehicles on the grid.

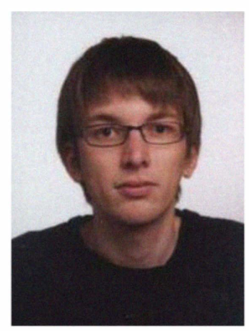

Jeroen Tant (S'08) received the M.Eng. degree in Electronics-ICT from the Hogeschool WestVlaanderen, Kortrijk, Belgium, in 2007 and the M.Sc. degree in Mathematical Engineering from the Katholieke Universiteit Leuven (K.U.Leuven), Leuven, Belgium, in 2009. He is currently working as a Research Assistant at K.U.Leuven with the division ESAT-ELECTA. His research topics include simulation and modelling of power electronic systems and deterministic and stochastic numerical methods for the analysis of power distribution grids.

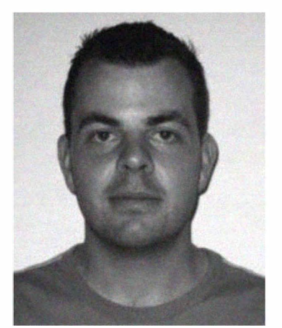

Edwin Haesen (S'05-M'09) received his M.Sc. and $\mathrm{Ph} . \mathrm{D}$. degrees in electrical engineering from the Katholieke Universiteit Leuven, Belgium, in 2004 and 2009. For his Master Thesis on Technical Aspects of Congestion Management he received the European Talent Award for Innovative Energy Systems 2005.

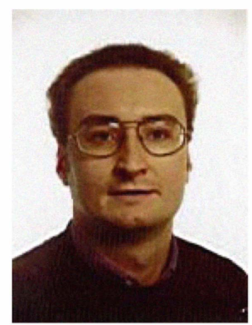

Johan Driesen (S'93-M'97) was born in 1973 in Belgium. He received the M.Sc. degree in 1996 as Electrotechnical Engineer from the K.U. Leuven, Belgium. He received the Ph.D. degree in Electrical Engineering at K.U.Leuven in 2000 on the finite element solution of coupled thermal-electromagnetic problems and related applications in electrical machines and drives, microsystems and power quality issues. Currently he is an associate professor at the K.U.Leuven and teaches power electronics and drives. In 2000-2001 he was a visiting researcher in the Imperial College of Science, Technology and Medicine, London, UK. In 2002 he was working at the University of California, Berkeley, USA. Currently he conducts research on distributed energy resources, including renewable energy systems, power electronics and its applications, for instance in renewable energy and electric vehicles.

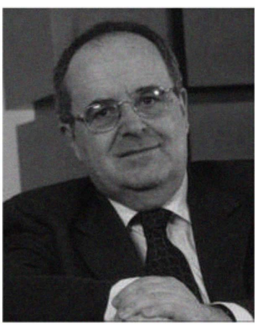

Ronnie Belmans (S'77-M'84-SM'89-F'05) received the M.S. degree in electrical engineering in 1979 and the $\mathrm{Ph} . \mathrm{D}$. degree in 1984, both from the K.U.Leuven, Belgium, the Special Doctorate in 1989 and the Habilitierung in 1993, both from the RWTH, Aachen, Germany. Currently, he is a full professor with the K.U.Leuven, teaching electric power and energy systems. His research interests include techno-economic aspects of power systems, power quality and distributed generation. He is also guest professor at Imperial College of Science, Medicine and Technology, London-UK. Since June 2002 he is chairman of the board of directors of ELIA, de Belgian transmission grid operator. 